Title

\title{
Correlation of Plasma Klotho Levels and Carotid Intima-Media Thickness in CKD Patients on Regular Hemodialysis
}

\section{Authors: \\ Radar Radius Tarigan, HR Lubis, AT Nasution \\ Nephrology-Hypertension Division, Internal Medicine Departement, \\ Faculty of Medicine Universitas Sumatera Utara}

Editor

Pringgodigdo Nugroho

Received 74 May 2019, revised 9 July 2019, accepted 29 November 2019, published 2 December 2019

\section{ABSTRACT}

Background: The incidence of mortality caused by cardiovascular disease (CVD) is 5-10 times higher in patients with chronic kidney disease on regular hemodialysis (CKD-HD) compared to the general population. In CKD patients there is a decrease in the distal tubular renal cells which are the main sources of Klotho in the body. The functions of Klotho includes maintaining endothelial integrity and permeability, and as an endogenous inhibitor of vascular calcification. Vascular calcification is a risk factor for CVD and CVD-related deaths. Previous studies reported an association between vascular calcification and Klotho plasma levels. One of the indicators for vascular calcification is carotid intima-media thickness (CIMT).

Objective: To determine the correlation of plasma Klotho levels with the CIMT in CKD-HD patients.

Method: This cross-sectional study was conducted at the Rasyida Kidney General Hospital in Medan from April to July 2018. Patients with CKD who have undergone routine hemodialysis are examined for plasma levels of Klotho and real-time ultrasound examinations to determine the IMT of the carotid artery. Data were 34 then analyzed with the Mann Whitney test, binominal logistic regression and Pearson correlation.

Results: Seventy subjects showed an average plasma Klotho level of $281.43+298.63 \mathrm{pg} / \mathrm{ml}$ with an average IMT of the carotid artery of $0.22+0.09 \mathrm{~cm}$. With the Pearson correlation test, the negative correlation of plasma Klotho levels with CIMT was statistically significant with weak correlation strength $(\mathrm{p}=0.002 ; \mathrm{r}$ $=-0.368$ ). Analysis was then followed by dividing the subjects into 2 groups, with CIMT $>1 \mathrm{~mm}(\mathrm{n}=39)$ and the CIMT $\leq 1 \mathrm{~mm}$ group $(\mathrm{n}=31)$. Mann-Whitney test was performed with the results that there were differences in plasma Klotho levels between CIMT groups which were statistically significant $(p=0.001)$. Secondary analysis of the data using the binominal logistic regression analysis test was performed on risk factors associated with CIMT and the results of Klotho serum levels, Diabetes Mellitus and Hypertension were found to be significant risk factors for increased CIMT ( $p$ $<0.05)$.

Conclusion: There is a weak correlation between plasma Klotho level and the IMT of the carotid artery in CKD-HD patients. Further analysis showed the level 
of Klotho significantly higher in patients with carotid IMT $>1 \mathrm{~mm}$ compared to carotid $\mathrm{IMT} \leq 1 \mathrm{~mm}$.

Keywords: plasma Klotho, intima-media thickness, carotid artery, chronic kidney disease

\section{Background}

Patients with CKD and CVD have higher mortality rate (58-71\%) compared with patients with CVD and normal renal function $(22-27,5 \%){ }^{1}$ Vascular calcification is initially thought as a passive process created by mineral deposition in patients with mineral imbalance. ${ }^{2}$ Under the physiological state, blood vessels and heart valves are protected from concentrated calcium and phosphate serum by its active inhibitors which protect from abnormal mineral deposition in soft tissue. Some inhibitors were found, such as pyrophosphate, adenosine, Gla matrix protein, osteopontin, fetuin-A, osteoprotegerin, bone morphogenetic protein-7, and Klotho. If instability of calcification inhibitors and calcification initiator concentration occur. Blood vessels will occur in the vascular wall or heart valves. ${ }^{3}$

In the beginning, Klotho is identified in mutant mice's strain that induces a lot of abnormalities that are similar to human's aging, including atherosclerosis, endothelial dysfunction, low bone mineral density, sarcopenia, skin atrophy, and cognitive impairment, and a shorter life span. ${ }^{4}$ There are two types of Klotho: membrane type and secreted type. Klotho membrane acts as an obligate coreceptor for FGF-23, hormone derived from bones that induce phosphate excretion into the urine. ${ }^{5}$ Secreted Klotho will be involved in the regulation of nitric oxide production in the endothelium, integrity maintenance and endothelial permeability, calcium homeostasis in renal and intracellular insulin inhibition and insulin-like growth factor-1 signaling. ${ }^{6}$

As a hormone, Klotho acts related to anti-aging and cardiovascular system protector. Klotho decreases oxidative stress by inhibiting insulin and insulin-like growth factor-1 signaling pathways. This increases nitric oxide endothelial production and therefore repairs endothelium vasodilatation. Klotho is vascular calcification endogen inhibitor, lower Klotho levels result in more artery calcifications than in higher Klotho levels.

Many studies have reported a correlation between the thickness of carotid intima-media with the occurrence and the severity of vascular calcification. It has also been proved that the state of the carotid artery wall reflects the condition of the coronary artery wall, so the thickening of carotid intima-media or the presence of plaque in the carotid artery can be used as an indicator for the occurrence of vascular calcification in heart blood vessels. There have been no data about the correlation of plasma Klotho levels with blood vessel calcification that is decided with carotid intima-media thickness in chronic kidney disease patients undergoing hemodialysis. This research aims to discover the correlation of plasma Klotho level with carotid intima-media thickness, correlation of plasma Klotho levels with $\mathrm{CKD}-\mathrm{MBD}$ marker ( $\mathrm{Ca}, \mathrm{P}$ and $\mathrm{Mg}$ ), risk factors affecting vascular calcification occurrence, correlation of plasma Klotho level with the age of chronic kidney disease patients with regular hemodialysis.

\section{Methods}

The design used in this study is cross-sectional with the independent variable is plasma Klotho levels and the dependent variable is carotid intima-media thickness in chronic kidney disease patients undergoing hemodialysis. This study was conducted from April to July 2018 at Rasyida Kidney General Hospital and in Faculty Medicine of Universitas Sumatera Utara Integrated Laboratorium.

The technique used to collect samples in this research 
Table 1. Patients' Demographic Characteristics

\begin{tabular}{|c|c|}
\hline Characteristics & $\mathrm{n}=70$ \\
\hline \multicolumn{2}{|l|}{ Gender, n (\%) } \\
\hline Male & $43(61.4)$ \\
\hline Female & $27(38.6)$ \\
\hline Age, average (SB), year & $54.21(10.86)$ \\
\hline Initial age undergoing HD, average (SB), year & $47.84(10.90)$ \\
\hline \multicolumn{2}{|l|}{ Frequency of HD, n (\%) } \\
\hline 10 hours/week & $50(71.4)$ \\
\hline 12 hours/week & $20(28.6)$ \\
\hline Period undergoing HD, average (SB), months & 71.30 (31.79) \\
\hline \multicolumn{2}{|l|}{ History of Diabetes, n (\%) } \\
\hline None & $49(70.0)$ \\
\hline Yes & $21(30.0)$ \\
\hline \multicolumn{2}{|l|}{ History of hypertension, n (\%) } \\
\hline None & 30 (42.9) \\
\hline Yes & $40(57.1)$ \\
\hline \multicolumn{2}{|l|}{ History of blood vessel and heart disease, n (\%) } \\
\hline None & $62(88.6)$ \\
\hline Yes & $8(11.4)$ \\
\hline \multicolumn{2}{|l|}{ History of stroke, n (\%) } \\
\hline None & $66(94.3)$ \\
\hline Yes & $4(5.7)$ \\
\hline \multicolumn{2}{|l|}{ History of smoking, $n$ (\%) } \\
\hline None & $47(67.1)$ \\
\hline Yes & $23(32.9)$ \\
\hline \multicolumn{2}{|c|}{$\begin{array}{l}\text { History of consuming calcium-based phosphate binder, } \\
n(\%)\end{array}$} \\
\hline & $33(47.1)$ \\
\hline \multicolumn{2}{|l|}{ None } \\
\hline & 37 (52.9) \\
\hline \multicolumn{2}{|l|}{ Yes } \\
\hline \multicolumn{2}{|c|}{$\begin{array}{l}\text { History of consuming calcium-free phosphate binder, } \mathrm{n} \\
\text { (\%) }\end{array}$} \\
\hline \multirow{2}{*}{\multicolumn{2}{|c|}{$59(84.3)$}} \\
\hline & \\
\hline & $11(15.7)$ \\
\hline \multicolumn{2}{|l|}{ Yes } \\
\hline \multicolumn{2}{|l|}{ Interpretation of carotid USG, n (\%) } \\
\hline Low CIMT ( $\leq 1 \mathrm{~mm})$ & $31(44.3)$ \\
\hline Increased CIMT (>1 mm) & $39(55.7)$ \\
\hline
\end{tabular}


is consecutive sampling which is choosing the samples based on inclusion and exclusion criteria until the amount of samples is fulfilled. The inclusion criteria are CKD patients who have undergone hemodialysis for at least 41 months. Regularly undergo hemodialysis 2-3 times a week. Willing to have blood samples drawn and undergo carotid artery sonography examination. The exclusion criteria are patients who cannot lie on their backs for ultrasonography (USG) examination, inadequate blood samples, and incomplete medical records. The total number of subjects needed for this study is 67 .

The independent variable is plasma klotho level (pg/ml) collected with the ELISA method measured with Bio Vendor equipment Catalogue Number RD 191037100. The observed variables are carotid intima-media thickness $(\mathrm{mm})$ obtained from real-time USG examination, carotid intima-media thickness obtained from the average thickness of two examinations. The confounding variables in this study include serum calcium level, serum phosphate level, age, the period of hemodialysis, and body mass index.

Data were analyzed using univariate analysis test to determine the characteristics of research subjects, bivariate analysis with hypothesis testing student's T-test with the alternative of Mann-Whitney, continued with Pearson correlation analysis with the alternative of Spearmen test. Multivariate test using binominal logistic regression. The analysis was performed using SPSS software version 22 .

\section{Results}

\section{Subjects' Baseline Characteristics}

This study was attended by 70 chronic kidney disease patients undergoing regular hemodialysis and have fulfilled the inclusion criteria (Table 1) which consisted of 43 male patients $(61.4 \%)$ and 27 female patients $(38.6 \%)$. The average age of the patients observed was InaKidney | Vol. II | Is. 3| Sept - Dec 2019
54.21 (10.86) years old with the average initial age undergoing hemodialysis is 47.84 (10.9) years old and the average of time undergoing hemodialysis was 71.30 (31.79) months. The majority of patients as many as $71.4 \%$ undergoing hemodialysis with the frequency of 10 hours/week with the most common ethnic group coming from Toba tribe with 28 patients $(40 \%)$, followed by Karo ethnic with 18 patients $(25.7 \%)$ and Melayu/Minang ethnic with 11 patients (15.7\%). Comorbidities in chronic kidney disease patients undergoing hemodialysis were hypertension in 40 patients $(57.1 \%)$, diabetes mellitus in 21 patients $(30 \%)$, heart disease in 8 patients $(11.4 \%)$ and stroke in 4 patients (5.7\%). There were 23 smoker patients $(32.9 \%)$ and 43 non-smoker patients (67.94\%). USG examination showed that as many as 39 patients $(55.7 \%)$ developed IMT thickness $>1 \mathrm{~mm}$.

In this study, the lab results characteristics showed that average serum calcium was $9.58(0.78) \mathrm{mg} / \mathrm{dl}$, the majority of patients $(87.1 \%)$ were in the range of 8.5-10.5 $\mathrm{mg} / \mathrm{dl}$ of serum calcium levels. Average phosphor serum was $5.46(0.60) \mathrm{mg} / \mathrm{dl}$ with the phosphor serum $>5.5$ $\mathrm{mg} / \mathrm{dl}$ group consisting of 34 people (48.6\%). Average haemoglobin, leukocytes, thrombocytes and magnesium levels were 9.93 (1.25) gr/dl, 10832 (1807.8)/ $\mathrm{mm}^{3}, 265614(60736.37) / \mathrm{ml}$, and $2.18(0.367) \mathrm{mg} / \mathrm{dl}$ respectively. For the average Klotho level in patients was 281.43 (298.63) pg/ml. (Table 2)

\section{Klotho levels and IMT of the carotid artery}

Analysis test with Chi-Square showed there was no correlation between gender, frequency of hemodialysis, heart comorbidities, and stroke with CIMT $(p=0.606 ; p=0.319 ; p=0.648 ; p=0.799 ; p=1 ; p=0.315)$. Meanwhile, there was a statistically significant correlation between diabetes mellitus and hypertension comorbidities with CIMT $(p=0.008 ; p=0.001)$ with ratio 
prevalence in diabetes mellitus was $\mathrm{PR}=5.216$ (1.53117.775) and hypertension was $\mathrm{PR}=5.273$ 14.748). (Table 3)

Table 2. Patients' Laboratorium Parameter

\begin{tabular}{|c|l|}
\hline Information & $\mathbf{n}=\mathbf{7 0}$ \\
\hline Serum Calsium, average (SB), mg/dL & $9.58 \pm 0.78$ \\
\hline$<8,5$ & 4 (5.7\%) \\
\hline $8,5-10,5$ & $61(87.1 \%)$ \\
\hline$>10,5$ & $5(7.1 \%)$ \\
\hline Serum Phosphor, average (SB), mg/dL & $5.46 \pm 0.60$ \\
\hline 3,55-55,5 & $36(51.4 \%)$ \\
\hline$>5,5$ & $34(48.6 \%)$ \\
\hline Ca X P, average (SB) & $53.37 \pm 8.61$ \\
\hline Hemoglobin, average (SB), gr/dL & $9.93 \pm 1.25$ \\
\hline Leukocytes, average (SB), $/ \mathrm{mm}{ }^{3}$ & $10832 \pm 1807.8)$ \\
\hline Magnesium, average (SB), $\mathrm{mg} / \mathrm{dL}$ & $2.18 \pm 0.367$ \\
\hline C-IMT, average (SB), cm & $24.21 \pm 4.64$ \\
\hline Thrombocytes, average (SB), /mL & $265614 \pm 60736.37$ \\
\hline S-Klotho, average (SB), pg/mL & $281.43 \pm 298.63)$ \\
\hline S- albumin (g/dL) & \\
\hline
\end{tabular}

Table 3 also demonstrates that the Mann-Whitney analysis test shows that there is no statistically significant difference in age, initial age of hemodialysis, BMI, Phosphor, Magnesium, Leukocytes, Thrombocytes, and Albumin levels CIMT $>1 \mathrm{~mm}$ and CIMT $\leq 1 \mathrm{~mm}$ group $(p=0.024 ; p=0.078 ; p=0.001)$.

Correlation between plasma Klotho levels with the thickening of carotid intima-media showed that there was a negative correlation between plasma Klotho towards the thickness of carotid intima-media which was statistically significant with weak strength correlation $(p=0.002 ; r=-0.368)$, figure 3 .

Plasma Klotho levels in IMT $>1 \mathrm{~mm}$ lower than in $\mathrm{IMT} \leq 1 \mathrm{~mm}$ group which are $187.38 \pm 97.93 \mathrm{pg} / \mathrm{ml}$ compared with $399.74 \pm 408.67 \mathrm{pg} / \mathrm{ml}$. There is a statistically significant difference in plasma Klotho levels between IMT $>1 \mathrm{~mm}$ group and $\mathrm{IMT} \leq 1 \mathrm{~mm}$ group $(p=0.001)$, figure 2 .

\section{Age and Klotho Levels}

Secondary analysis of Klotho levels between non-geriatric patients $(<65$ years old) and geriatric patients $(\geq$ 65 years old) using independent T-test showed statistical significance, where Klotho levels are lower in older patients $(p=0.002)$.

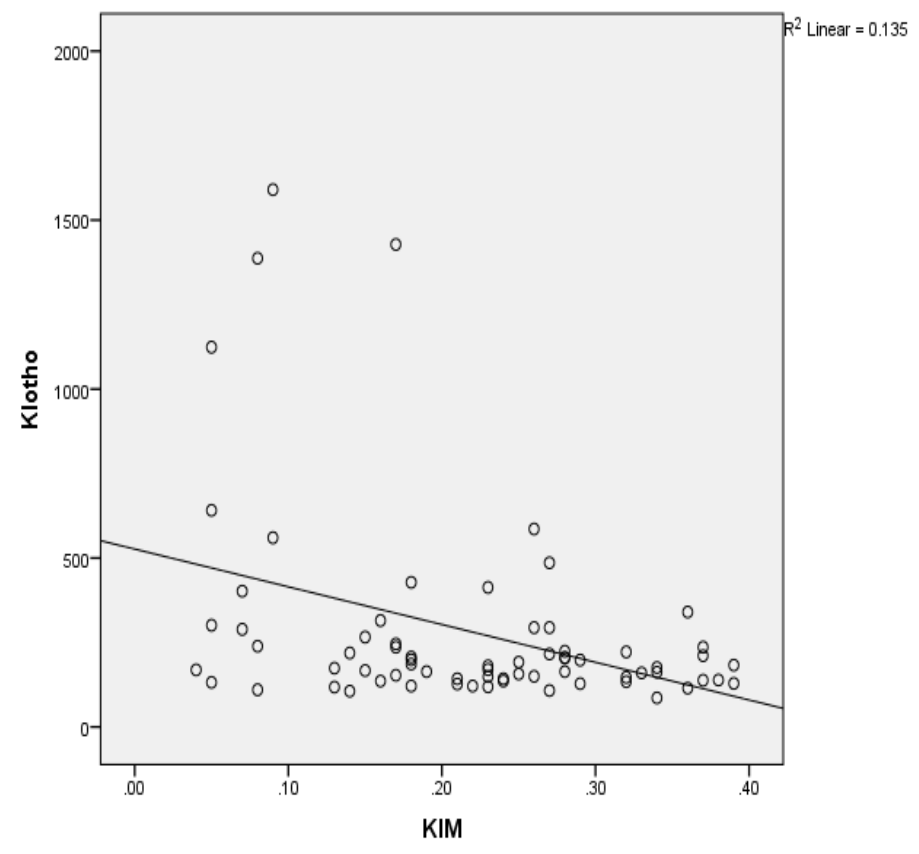

Figure 1. Correlation between plasma Klotho levels with carotid intima-media thickness

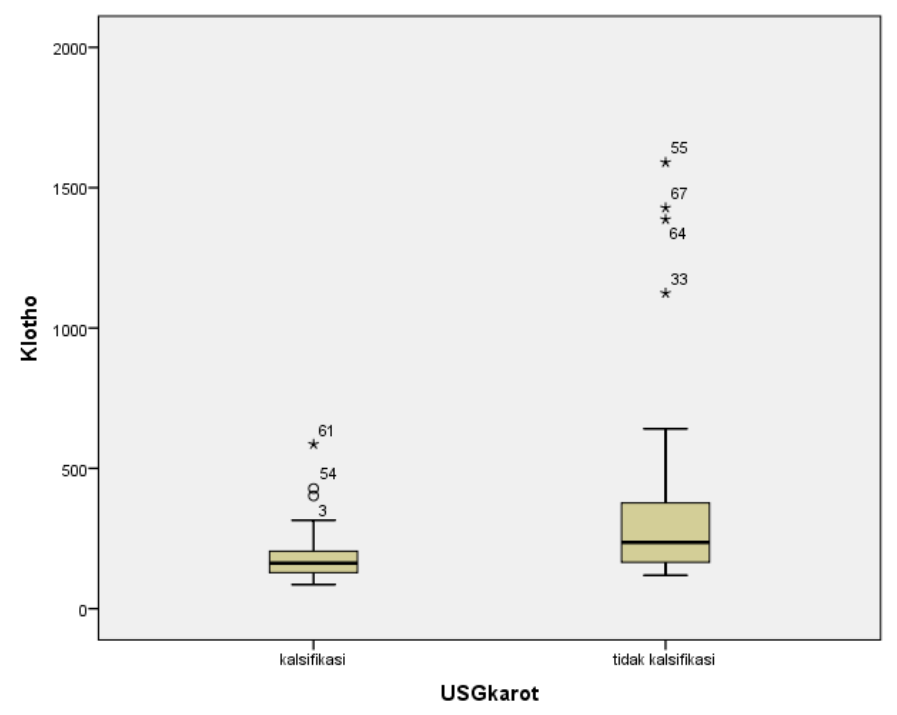

Figure 2. Boxplot Diagram the comparison of Klotho levels between IMT $>1 \mathrm{~mm}$ group with $\mathrm{IMT} \leq 1 \mathrm{~mm}$ group. 
Table 3. Basic characteristics risk factors for the thickening of carotid artery intima-media.

\begin{tabular}{|c|c|c|c|c|c|}
\hline Characteristics & $\begin{array}{l}\text { IMT > } 1 \mathrm{~mm} \\
(\mathrm{n}=39)\end{array}$ & $\begin{array}{l}\mathrm{IMT} \leq 1 \mathrm{~mm} \\
(\mathrm{n}=31)\end{array}$ & PR & $\mathrm{Cl}(95 \%)$ & p \\
\hline Gender $^{\mathrm{a}}$ & & & 1.29 & $0.490-3.395$ & 0.606 \\
\hline Males & $25(64.1)$ & $18(58.1)$ & & & \\
\hline Females & $14(35.9)$ & 13(41.9) & & & \\
\hline Age $^{b}$ & 54.18(10.99) & $54.26(10.87)$ & 1.001 & 0.958-1.045 & 0.972 \\
\hline $\begin{array}{l}\text { Initial Age undergoing } \\
H D^{b}\end{array}$ & $47.62 \pm 11.2$ & $48.13 \pm 10.69$ & 1.004 & $0.962-1.049$ & 0.745 \\
\hline Period of $H D^{b}$ & $73.18(34.86)$ & $68.94(27.85)$ & 0.996 & 0.980-1.011 & 0.623 \\
\hline Frequency of $H D^{a}$ & & & 0.783 & $0.273-2.244$ & 0.648 \\
\hline 10 hours/week & $27(69.2)$ & $23(74.2)$ & & & \\
\hline 12 hours/week & $12(30.8)$ & $8(25.8)$ & & & \\
\hline \multicolumn{6}{|l|}{ Comorbidities $^{a}$} \\
\hline Diabetes & $17(43.6)$ & $4(12.9)$ & 5.216 & $1.531-17.775$ & 0.008 \\
\hline Hypertension & $29(74.4)$ & $11(35.5)$ & 5.273 & $1.885-14.748$ & 0.001 \\
\hline $\mathrm{CHD}$ & $5(12.8)$ & $3(9.7)$ & 1.373 & $0.301-6.252$ & 1 \\
\hline Stroke & $1(2.6)$ & $3(9.7)$ & 0.246 & $0.024-2.487$ & 0.315 \\
\hline Smokinga & $12(30.8)$ & $11(35.5)$ & 0.808 & $0.297-2.201$ & 0.799 \\
\hline $\begin{array}{l}\text { Calcium-based phos- } \\
\text { phate binder }\end{array}$ & $20(51.3)$ & $17(54.8)$ & 0.867 & $0.337-2.232$ & 0.478 \\
\hline $\begin{array}{l}\text { Calcium-free phosphate } \\
\text { binder }^{\mathrm{a}}\end{array}$ & $6(15.4)$ & $5(16.1)$ & 0.945 & $0.259-3.446$ & 1 \\
\hline $\mathrm{BMI}^{\mathrm{b}}$ & $24.73 \pm 4.66$ & $23.55 \pm 4.60$ & 0.942 & $0.842-1.054$ & 0.269 \\
\hline \multicolumn{6}{|l|}{ Laboratorium Results } \\
\hline Calcium & $9.78 \pm 0.742$ & $9.32 \pm 0.78$ & 0.458 & $0.240-0.874$ & 0.024 \\
\hline Phosphor & $5.52 \pm 0.61$ & $5.39 \pm 0.58$ & 0.699 & $0.314-1.558$ & 0.441 \\
\hline$C A \times P$ & $54.2 \pm 8.78$ & $52.30 \pm 8.42$ & 0.974 & $0.921-1.030$ & 0.269 \\
\hline Magnesium & $2.23 \pm 0.43$ & $2.12 \pm 0.26$ & 0.417 & $0.097-1.788$ & 0.414 \\
\hline Hemoglobin & $10.18 \pm 1.34$ & $9.61 \pm 1.07$ & 0.679 & $0.452-1.020$ & 0.078 \\
\hline Leukocytes & $\begin{array}{l}11056.41 \pm \\
1894.84\end{array}$ & $10551.61 \pm 1680.05$ & 1 & 1 & 0.168 \\
\hline Thrombocytes & $\begin{array}{l}270102.56 \pm \\
64090.31\end{array}$ & $\begin{array}{l}259967.74 \pm \\
56766.47\end{array}$ & 1 & 1 & 0.566 \\
\hline S-Klotho & $187.38 \pm 97.93$ & $399.74 \pm 408.67$ & 1.005 & $1.001-1.010$ & 0.001 \\
\hline S- albumin & $3.96 \pm 0.31$ & $3.87 \pm 0.32$ & 0.406 & $0.088-1.865$ & 0.446 \\
\hline
\end{tabular}




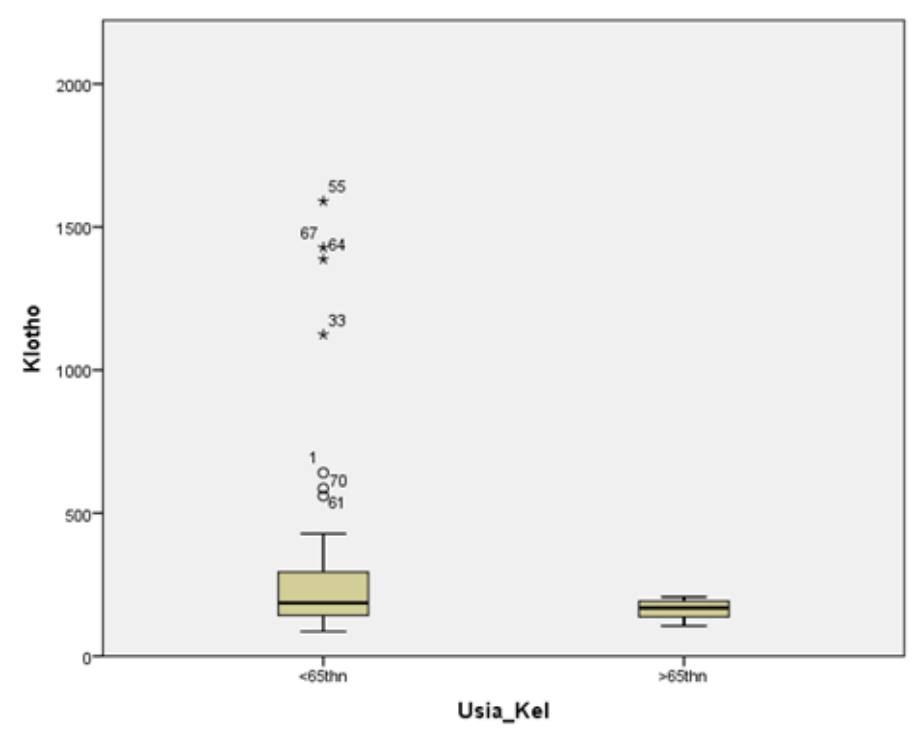

Figure 3. Boxplot Diagram the comparison of Klotho levels between subjects $\geq 65$ year old group with $65<$ year old group

\section{Risk Factors for Increased CIMT}

Binominal logistic regression test analysis was performed towards the associated risk factors from the bivariate test $(\mathrm{p}<0.25)$ with thickening of IM and the result obtained was the risk factors that affected IMT were plasma Klotho levels, diabetes mellitus, and hypertension $(\mathrm{p}<0.05)$. The low plasma Klotho levels $(<396.3$ $\mathrm{pg} / \mathrm{ml}$ ) are associated with increased CIMT. History of diabetes and hypertension will also increase the risk of the thickening of the carotid artery intima-media.

Based on statistical analysis, formulation to predict the thickening of the carotid artery intima media is

$(-1.058)+(0.005 \times$ klotho levels $)+(1.422 \mathrm{x}$ diabetes $)+(1.463 \mathrm{x}$ hypertension $)$

Klotho levels : $0=$ normal, $1=$ high. Diabetes : $0=$ non diabetes, $1=$ overt diabetes. Hypertension: $0=$ normotension, 1 = history of hypertension.

Table 4. Multivariate analysis of risk factors associated 40 with the thickening of the carotid artery intima-media.

\begin{tabular}{llll}
\hline $\begin{array}{l}\text { Characteris- } \\
\text { tics }\end{array}$ & PR & Cl (95\%) & p \\
& & & \\
Diabetes & 4.147 & $1.075-15.993$ & 0.039 \\
Hypertension & 4.318 & $1.373-13.584$ & 0.012 \\
S-Klotho & 1.005 & $1.001-1.011$ & 0.046 \\
Hemoglobin & 0.924 & $0.543-1.574$ & 0.772 \\
Leukocytes & 1 & 1 & 0.353 \\
Calcium & 0.568 & $0.247-1.305$ & 0.183 \\
\hline
\end{tabular}

\section{Correlation between plasma Klotho levels with se-} rum Phosphor, Calcium, Magnesium levels

Correlation test between plasma Klotho levels with mineral metabolism marker such as serum Phosphor, Calcium, and Magnesium by using Pearson Correlation test showed that there was a negative correlation between plasma Klotho levels with serum Phosphor, Calcium, Statistically insignificant Magnesium levels $(p=0.303 ; p=0.116 ; p=0.110)$, figure 4 .

\section{Discussion}

In this study, it was discovered that plasma Klotho levels were significantly lower in chronic kidney disease patients undergoing regular hemodialysis with older age, but this decrease never reached zero. This finding showed that plasma Klotho is not just expressed in renal. Kinoshita (2016) stated that Klotho is especially expressed in renal cells (mainly in distal convoluted tubule), brain, reproduction organ, pituitary gland, parathyroid gland, urinary bladder, skeletal muscle, placenta, thyroid gland, and colon. ${ }^{6}$ 

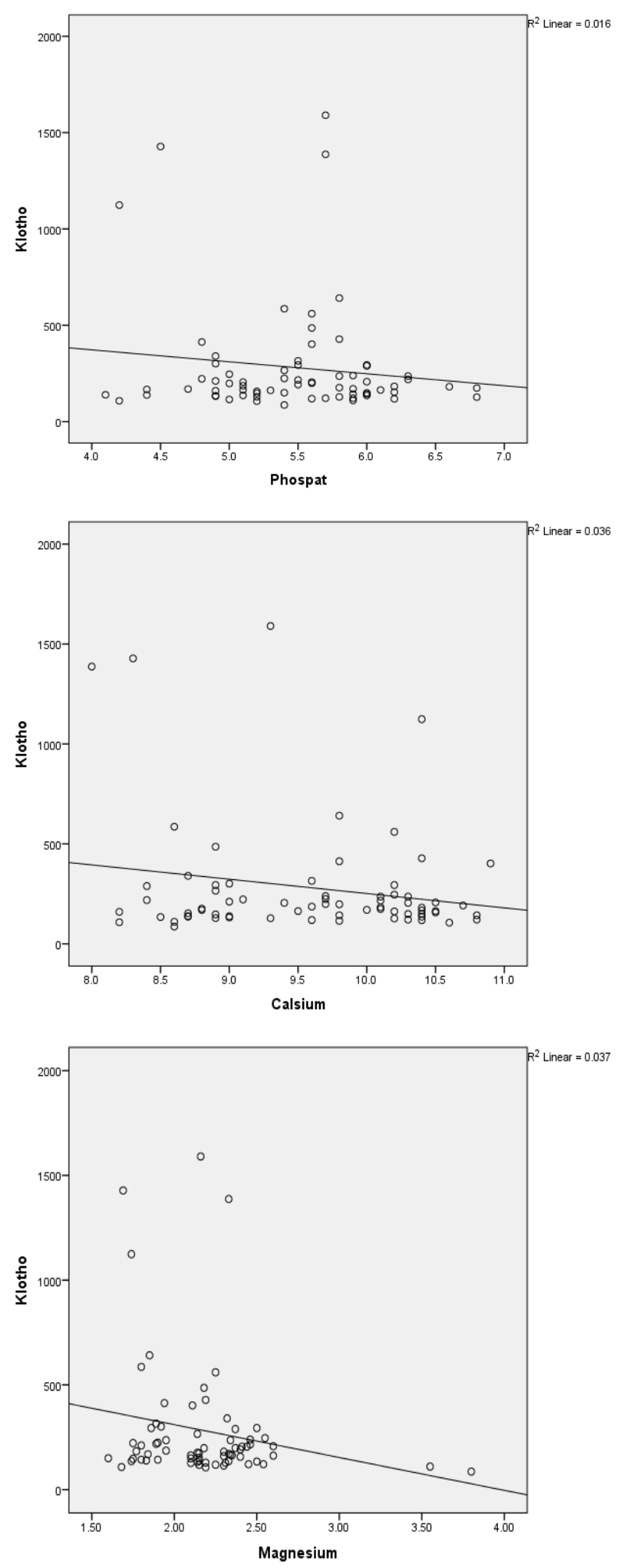

Figure 4. Correlation between plasma Klotho levels with serum Phosphor (a), serum Calcium (b), and serum Magnesium (c) levels.

The alteration of age-related plasma Klotho levels is often related especially to CKD patients. Research by Kitagawa et al showed that plasma Klotho levels de- creased as the age increased, and this result was statistically significant $(p=0.0034) .{ }^{7}$ Lindberg et al reported that there was a significant negative correlation between plasma Klotho and age. ${ }^{8}$ This study showed that patients with lower plasma Klotho levels were found in $\mathrm{CKD}$ patients undergoing regular hemodialysis with older age.

Endothelial dysfunction is a systemic pathological condition caused by an imbalance between vasorelaxation and vasoconstriction of blood vessels. This imbalance is mainly a result of the decreased NO bioavailability and/or the increased reactive oxygen species generation. Inflammatory stimulation and hypertension accelerate endothelial dysfunction. Plasma Klotho levels are related to the presence of vascular dysfunction such as arterial stiffness in CKD patients.

Klotho can induce NO production or shows an anti-inflammatory role in protecting the endothelium. Existing studies reported that low plasma Klotho levels are associated significantly with cardiovascular disease markers such as the increased thickness of carotid intima-media, coronary artery disease, and left ventricle dysfunction. ${ }^{9}$ Plasma Klotho level is found significantly correlated with CKD-MBD marker which is an independent arterial stiffness biomarker in CKD patients. ${ }^{7}$ This study showed that patients with lower plasma Klotho levels were more often related to the thickening of carotid intima-media. This result is in accordance with a finding by Keles et al that showed that the group with lower plasma Klotho levels had a greater thickness of tunica intima compared with the group with higher plasma Klotho levels, and this discovery was statistically significant $(\mathrm{p}<0.001) .{ }^{10}$

The role of plasma Klotho in atherosclerosis development in CKD patients undergoing hemodialysis is not the only triggering factor, there are other factors including obesity, diabetes mellitus, hypertension, dyslipidemia, and smoking. These are the possible expla- 
nation for the difference between data researched on experimental humans and the decreased plasma Klotho in CKD patients with varieties of comorbidities.

Secondary analysis of this study showed that there was a negative correlation between plasma Klotho levels with serum Phosphor, Magnesium and Calcium levels. Furthermore, this research also revealed that lower plasma Klotho levels were significantly related to CVD marker such as the increased thickness of carotid intima-media. The result of this study has demonstrated that plasma Klotho levels started to decrease in mild CKD patients (stadium $\leq 2$ ) and preceded the increase of serum FGF-23, PTH, and Phosphate, this shows that Klotho might be a sensitive biomarker on decreasing renal function. ${ }^{3}$

This study has several limitations. We researched on only a small amount of samples. Data about calcium, phosphor dietary and drug intake that affected serum Phosphor, Calcium and Klotho levels were not collected. Although low Klotho levels were proved as stimulators in vascular calcification occurrence, the causality between Klotho and vascular calcification cannot be determined with certainty because this study uses a cross-sectional design with consecutive sampling as the method for collecting samples, in which this is weak in research. Therefore, it is advised to do further research with case-control or cohort design with larger samples.

In this study, carotid artery USG examination was performed by a radiologist, for further research, it is recommended to be conducted by two radiologists for comparison to determine the thickness of carotid intima-media thickness. Although the diagnostic value of plasma Klotho levels towards vascular calcification is relatively high, it cannot be ensured that the influence it has is direct or indirect through other mediators such as decreasing FGF-23, Fetuin-a, and vitamin D levels. Because of that reason, it is recommended to do further research with a more comprehensive examination to decide which factors are the most dominant in affecting vascular calcification.

\section{Conclusion}

Although the correlation was found to be weak, further analysis showed there's a significant difference between plasma Klotho levels between patients with CIMT $>1 \mathrm{~mm}$ and CIMT $\leq 1 \mathrm{~mm}$. Secondary analysis showed Klotho levels are lower in older patients.

\section{References}

1. Gansevoort RT, Correa-Rotter R, Hemmelgarn BR, Jafar TH, Heerspink HJ, Mann JF, et al. Chronic Kidney Disease and Cardiovascular Risk: Epidemiology, Mechanisms, and Prevention. Lancet. 2013 Jul 27;382(9889):339-52

2. Vervloet MG, Adema AY, Larsson TE, and Massy ZA. 2014. "The Role of Klotho on Vascular Calcification and Endothelial Function in Chronic Kidney Disease." Semin Nephrol. 2014 Nov;34(6):578-85. doi: 10.1016/j.semnephrol.2014.09.003.

3. Yamada S, Giachelli CM. Vascular Calcification in CKD-MBD: Roles for Phosphate, FGF23, and Klotho. Bone. 2017 Jul;100:87-93. doi: 10.1016/j.bone.2016.11.012

4. Xu Y, Sun Z. Molecular basis of Klotho: from gene to function in aging. Endocr Rev. 2015 Apr;36(2):174-93.

5. Guitierrez OM, Fibroblast Growth Factor 23, Klotho, and Disordered Mineral Metabolism in Chronic Kidney Disease: Unraveling the Intricate Tapestry of Events and Implication for Therapy. J Ren Nutr. 2013 May; 23(3): 250254. 